\title{
A frequent somatic mutation in the 3'UTR of GAPDH facilitates the development of ovarian cancer by creating a miR-125b binding site
}

\author{
PEISEN LIU ${ }^{1 *}$, YUMIN ZHONG $^{1 *}$, TING CAO $^{1}$, XIUJIE SHENG $^{1}$ and HUANG HUANG ${ }^{1-3}$ \\ ${ }^{1}$ Key Laboratory for Major Obstetric Diseases of Guangdong Province, The Third Affiliated Hospital, \\ Guangzhou Medical University; ${ }^{2}$ Lin He's Academician Workstation of New Medicine and \\ Clinical Translation at The Third Affiliated Hospital, Guangzhou Medical University, Guangzhou, \\ Guangdong 510150; ${ }^{3}$ Genex Health Company, Ltd., Beijing 100195, P.R. China
}

Received July 30, 2019; Accepted April 23, 2020

DOI: 10.3892/or.2020.7663

\begin{abstract}
Ovarian cancer (OVCA) is one of the most common types of cancer in women worldwide. Recent studies have focused on the presence and effect of somatic mutations in patients with OVCA; however, studies on the roles of mutations located in the untranslated regions (UTR) of genes in OVCA remain limited. In the present study, a frequent somatic mutation in the glyceraldehyde 3-phosphate dehydrogenase (GADPH) 3'UTR was identified using transcriptome sequencing of 120 pairs of OVCA tissue samples. The mutant GAPDH 3'UTR promoted tumor growth and cell motility. Furthermore, the mutation in the GAPDH 3'UTR significantly downregulated the levels of mature miR-125b by creating a new miR-125b binding site. Finally, STAT3 levels were increased in SKOV3 cells stably expressing the mutant GADPH 3'UTR, which is a critical target gene of miR-125b. In conclusion, the present study demonstrated that the mutation located in GAPDH 3'UTR promoted OVCA growth and development by sponging miR-125b and thereby affecting STAT3 expression levels.
\end{abstract}

\section{Introduction}

Ovarian cancer (OVCA) is one of the leading causes of cancer-associated deaths among women $(1,2)$. According to the World Health Organization, OVCA accounts for 150,000 deaths annually worldwide (3). Therefore, there is an urgent

Correspondence to: Dr Huang Huang, Key Laboratory for Major Obstetric Diseases of Guangdong Province, The Third Affiliated Hospital, Guangzhou Medical University, Guangzhou, Guangdong 510150, P.R. China

E-mail: huanghuang8011@163.com

*Contributed equally

Key words: GAPDH, somatic mutation, 3'untranslated region, ovarian cancer, miR-125b need to better understand the molecular mechanisms of tumor growth and development of OVCA.

Glyceraldehyde 3-phosphate dehydrogenase (GAPDH) is a key regulatory enzyme, which catalyzes the oxidative phosphorylation of glycerol-3-phosphate during glycolysis. It is one of the most extensively investigated housekeeping genes, and is widely used as an internal control for the analysis of gene expression levels. However, mounting evidence now recognizes GAPDH as a multifunctional protein involved in a range of biological processes, including membrane fusion and transport (4,5), apoptosis (6), DNA repair (7), DNA replication and regulation of transcription and translation (8). GAPDH expression is modulated by serum, epidermal growth factor and retinoic acid $(9,10)$. In addition, it has been reported that miRNA-mediated regulation is partly responsible for the variability in GAPDH expression (11). Recent studies have demonstrated that GAPDH expression is significantly upregulated in several types of cancer, such as renal $(12,13)$, breast (14) and nasopharyngeal cancer (15). A dysregulation of GAPDH expression may potentially be associated with tumorigenesis and malignancy.

Recently, studies have suggested that the genomes of the majority of cancers in adults contain thousands of somatic mutations (16), and mutations in the 3'untranslated region (3'UTR) may mediate oncogene activation or tumor suppressor inactivation by altering microRNA (miRNA/miR)-binding efficiency $(17,18)$. miRNAs are small, endogenous and non-coding RNAs that negatively regulate gene expression at the post-transcriptional level, by binding to the 3'UTR of their target mRNAs (19). It has also been reported that mRNAs, long non-coding RNAs (lncRNAs), and transcribed pseudogenes can act as competitive endogenous RNAs (ceRNAs) which co-regulate each other's expression by competing for shared microRNAs $(20,21)$, and the 3'UTR of mRNA alone can act as a ceRNA (22-25). Dysregulation of the expression of various miRNAs has been identified in several types of cancer, and have been demonstrated to contribute to the malignancy and tumorigenesis of cancer (26-28).

In the present study, it was demonstrated that the expression of GAPDH mRNA was upregulated in OVCA tissues, and 
a somatic mutation in the 3'UTR of GAPDH was identified, which was located in the seed sequence of miR-125b. The mutant GAPDH 3'UTR promoted tumor growth and cell motility by directly binding to miR-125b, thus upregulating the expression of the miR-125b target gene, STAT3, in OVCA.

\section{Materials and methods}

Clinical tissue specimens and cell lines. All experimental protocols in the present study were approved by The Third Affiliated Hospital of Guangzhou Medical University (Guangzhou, Guangdong, China). Between January 2015 and 2019, a total of 120 patients who were pathologically diagnosed with OVCA were enrolled in this study. The inclusion criteria were as follows: OVCA patients who were scheduled for cytoreductive surgery; patients aged above 18 years. Any patient who was in pregnancy, or who was diagnosed with a malignant tumor of another organ and tissue was excluded. The clinicopathological characteristics of these 120 OVCA patients are documented in Table I. All patients included in this study provided written informed consent prior to surgery. The cell lines used in this study were, SKOV3, OVCAR3 and 293T cells, and were all obtained from the American Type Culture Collection. 293T cells were cultured in DMEM (Gibco; Thermo Fisher Scientific, Inc.) containing 10\% FBS (Gibco; Thermo Fisher Scientific, Inc.) in a humidified incubator with $5 \% \mathrm{CO}_{2}$ at $37^{\circ} \mathrm{C}$. SKOV3 and OVCAR3 cells were cultured in RPMI-1640 medium with $10 \%$ FBS under the same incubation conditions.

Transcriptome library preparation and sequencing. Total RNA was extracted from cancer and normal tissues using TRIzol $^{\circledR}$ (Tiangen Biotech Co., Ltd.). RNA degradation and contamination were assessed by running a sample on a $1 \%$ agarose gel. RNA purity and concentration were detected using a NanoPhotometer ${ }^{\circledast}$ spectrophotometer (Implen $\mathrm{GmbH}$ ) and a Qubit $^{\circledR}$ RNA Assay kit with a Qubit ${ }^{\circledR}$ 2.0 Flurometer (Thermo Fisher Scientific, Inc.), respectively. RNA integrity was assessed using an RNA Nano 6000 Assay kit with a Bioanalyzer 2100 system (Agilent Technologies, Inc.). Subsequently, a total of $3 \mu \mathrm{g}$ of RNA from each sample was used to construct the Illumina sequencing library with a NEBNext ${ }^{\circledR}$ Ultra $^{\mathrm{TM}}$ RNA Library Prep kit for Illumina ${ }^{\circledR}$ (New England BioLabs, Inc.). The index codes were added to attribute sequences to each sample using a TruSeq PE Cluster kit v3-cBot-HS (Illumina, Inc.), and the prepared libraries were sequenced using an Illumina Hiseq ${ }^{\circledR}$ 2500 platform (Illumina, Inc.).

Generation of a stable cell line. The primer sequences for the amplification of GAPDH 3'UTR-WT or the mutant (CCTAG to CCTCAG) were as follows: GAPDH 3'UTR forward, 5'-CCA GCAAGAGCACAAGAGGA-3' and reverse, 5'-TGGTTG AGCACAGGGTACTT-3'. GAPDH 3'UTR-MUT (CCTAG to CCTCAG) and control DNA fragments were cloned into a pLKO.1-EGFP lentivirus vector. 293T cells were used for generation of lentiviral particles by transfecting the expression vectors and package vectors with PEI. A total of $72 \mathrm{~h}$ after transfection, supernatants containing the lentiviral particles were harvested, and the remaining cells were removed by concentration. For lentiviral infection, SKOV3 and OVCAR3 cells were seeded 1 day prior to
Table I. Clinicopathogical characteristics of the OVCA patients $(\mathrm{N}=120)$.

\begin{tabular}{lc}
\hline Clinicopathological characteristics & Data \\
\hline Age (years) & \\
Median age (years) & 54 \\
$\geq 60, \mathrm{n}(\%)$ & $54(45.00)$ \\
$<60, \mathrm{n}(\%)$ & $66(55.00)$ \\
BMI, kg/m² & \\
Mean (range) & $21.34(18.9-28.6)$ \\
FIGO stage, $\mathrm{n}(\%)$ & \\
I-II & $74(61.67)$ \\
III-IV & $46(38.33)$ \\
Histological type, $\mathrm{n}(\%)$ & \\
Serous & $71(59.17)$ \\
Clear cell & $12(10.00)$ \\
Mucinous & $17(14.16)$ \\
Endometrioid & $20(16.67)$
\end{tabular}

OVCA, ovarian cancer; BMI, body mass index; FIGO, International Federation of Gynecology and Obstetrics.

infection, and cells were infected with a multiplicity of infection (MOI) of 20 and 30, respectively. The lentivirus was diluted in RPMI-1640 medium containing Polybrene $(10 \mu \mathrm{g} / \mathrm{ml})$ and added to the cells for $24 \mathrm{~h}$ at $37^{\circ} \mathrm{C}$. Subsequently, the virus-containing medium was replaced with fresh supplemented RPMI-1640 medium, and the successfully infected cells were isolated using FACS based on eGFP fluorescence.

RNA transfection. miR-125b mimics, Ago2 small interfering (si)RNA and their corresponding negative controls (NCs) were purchased form Shanghai GenePharma Co., Ltd. Transfection was performed using Lipofectamine 3000 (Invitrogen; Thermo Fisher Scientific, Inc.). miR-125b mimics, Ago2 siRNA and $\mathrm{NC}$ at a final concentration of $50 \mathrm{nM}$ were used for transfection. The sequences of the Ago2 siRNAs used were as follows: Ago2-siRNA-1, 5'-GCCAGTAATCGAGTTTGTT-3'; Ago2-siRNA-2, 5'-GGTACCACCTGGTGGATAA-3'; and Ago2-siRNA-3, 5'-GCAGAAACACACCTACCTT-3'.

RNA extraction and reverse transcription-quantitative PCR. TRIzol ${ }^{\circledR}$ reagent (Tiangen Biotech Co., Ltd.) was used to isolate total RNA. A total of $2 \mu \mathrm{g}$ of each RNA sample was reverse transcribed into cDNA using FastQuant RT Super mix (Tiangen Biotech Co., Ltd.). qPCR was performed on a Bio-Rad MiniOpticon Real-Time PCR system (Bio-Rad Laboratories, Inc.) using a SYBR Green PCR Master mix (Tiangen Biotech Co., Ltd.). A Bugle-Loop ${ }^{\mathrm{TM}}$ miRNA qPCR kit (Guangzhou RiboBio Co., Ltd.) was used to quantify miR-125b and U6. Hsa-mir-125b-1_1_PR miScript Precursor Assay (Qiagen $\mathrm{GmbH}$ ) was used for quantification of precursor miR-125b. The primer sequences were as follows: GAPDH forward, 5'-AGCCACAATCGCTCAGACAC-3' and reverse, 5'-GCCCAATACGACCAAATCC-3'; Ago2 forward, 5'-CCT CCCATGTTTACAAGTCG-3' and reverse, 5'-TCTTTGTCC 

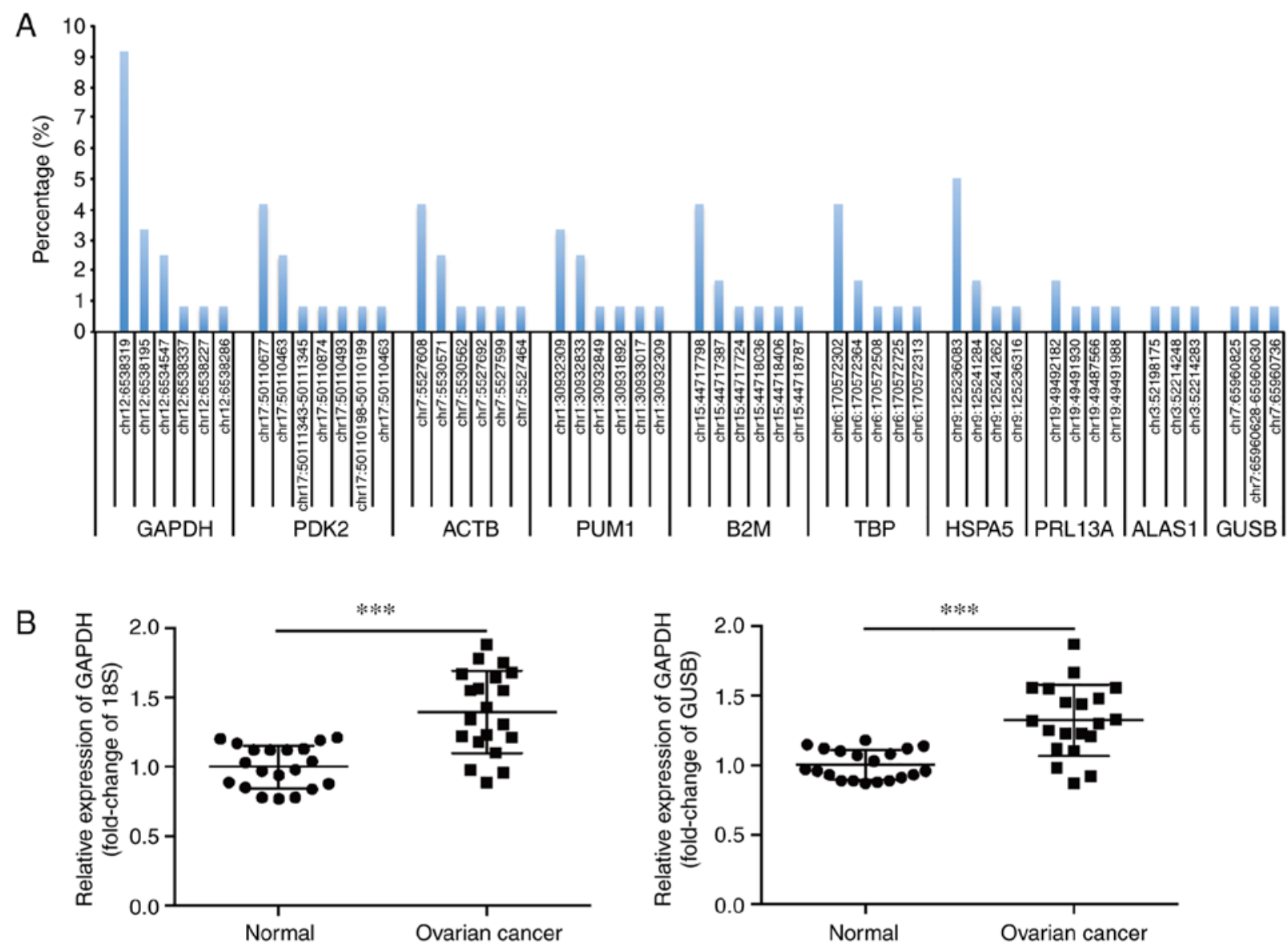

Figure 1. Identification of a frequent somatic mutation in the 3'UTR of GAPDH. (A) The top 10 housekeeping genes with high mutation rates in the UTR, as determined by transcriptome sequencing of 120 pairs of human OVCA tissues and the matching adjacent normal tissues. (B) Relative mRNA expression levels of GAPDH in 20 pairs of OVCA tissues and the matching adjacent normal tissues, with $18 \mathrm{~S}$ or GUSB as the internal control, respectively. ${ }^{* * *} \mathrm{P}<0.001$. UTR, untranslated region; OVCA, ovarian cancer; GAPDH, glyceraldehyde 3-phosphate dehydrogenase, PDK2, pyruvate dehydrogenase kinase 2; ACTB, $\beta$ actin; PUM1, Pumilio RNA binding family member 1; B2M, $\beta$-2-microglobulin; TBP, TATA-box binding protein; HSPA5, heat shock protein family A (Hsp70) member 5; RPL13A, ribosomal protein L13a; ALAS1, 5'-aminolevulinate synthase 1; GUSB, glucuronidase $\beta$.

TGCCACAATG-3'; STAT3 forward, 5'-CATATGCGGCCA GCAAAGAA-3' and reverse, 5'-ATACCTGCTCTGAAG AAACT-3'; GUSB forward, 5'-AGCGTGGAGCAAGACAG TG-3' and reverse, 5'-TCTGCATAGGGGTAGTGGCT-3'; and Pri-miR-125b forward, 5'-TGAACCTCGAACAGAAATTG CC-3' and reverse, 5'-TCCACCAAATTTCCAGGATGC-3'.

Western blotting. Total cellular proteins were solubilized in RIPA lysis buffer containing protease inhibitor cocktail (Sigma-Aldrich; Merck KGaA) and $1 \mathrm{mM}$ PMSF. Proteins were loaded on a $10 \%$ SDS gel, resolved using SDS-PAGE and subsequently transferred electrophoretically to a PVDF membrane (EMD Millipore). The membranes were blocked in 5\% non-fat-dried milk at room temperature for 30-60 min, and subsequently incubated with primary antibodies overnight at $4^{\circ} \mathrm{C}$ at the following dilutions: GAPDH (Santa Cruz Biotechnology, Inc.; cat. no. sc-25778) at 1:1,000, Ago2 (Abcam; cat. no. ab57113) at 1:2,000, STAT3 (Santa Cruz Biotechnology, Inc. cat. no. sc-8019) at 1:300, anti-human $\beta$-actin (Cell Signaling Technology, Inc.; cat. no. 4970) at 1:1,000. Subsequently, the membranes were washed and incubated for $1 \mathrm{~h}$ at room temperature with horseradish peroxidase-conjugated secondary antibodies (Cell Signaling Technology, Inc.; cat. no. 7074) at a dilution of 1:2,000.
Following several washes, signals were visualized using an ECL system (Tiangen Biotech Co., Ltd.). $\beta$-actin was used as the loading control. Western blot protein grayscale values were detected and analyzed by Image $(1.48 \mathrm{~V} ; \mathrm{NIH})$.

Luciferase assay. For the luciferase assays, the WT and mutant GAPDH 3'UTR, and the WT and mutant (CTCAGGGA to CTACTAGA) STAT3 3'UTR were amplified by PCR. The primer sequences for the amplification of STAT3 3'UTR-WT and STAT3 3'UTR-MUT were as follows: STAT3 3'UTR forward, 5'-ACCTGGCCTTTGGTGTTGAA-3' and reverse: 5'-GCTGAGGCAAGGTGGTTTTG-3'. The DNA fragments were cloned into a pGL3 plasmid 3 ' to the firefly luciferase gene. A total of $4 \times 10^{4} 293 \mathrm{~T}$ cells were co-transfected with $200 \mathrm{ng}$ of microRNA mimics, $200 \mathrm{ng}$ of one of the pGL3 firefly luciferase constructs and $20 \mathrm{ng}$ of pGL3 Renilla luciferase construct, which was used as the normalization control. After $48 \mathrm{~h}$, cells were lysed and luciferase activity was measured using a Dual-Luciferase Reporter Assay System (Promega Corporation), according to the manufacturer's protocol.

Cell Counting Kit-8 (CCK-8) assay. Cells were seeded in 96-well plates at a density of $2 \times 10^{3}$ cells/well in $100 \mu \mathrm{l}$ culture medium. A total of $10 \mu \mathrm{l}$ CCK-8 reagent (Beijing Transgen 
A

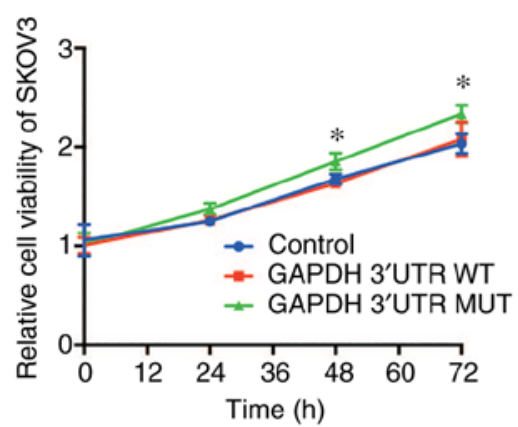

B

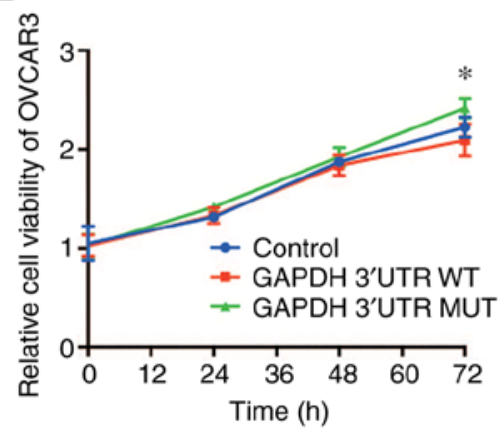

$\mathrm{E}$

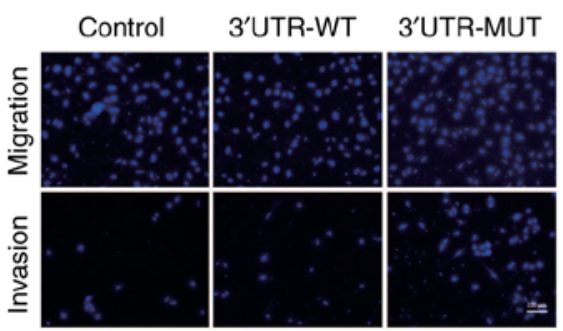

C

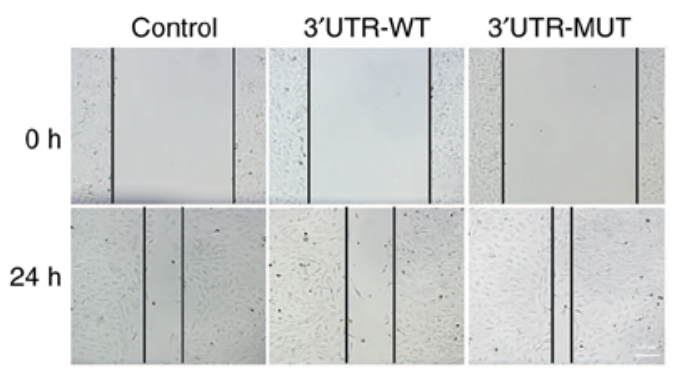

D
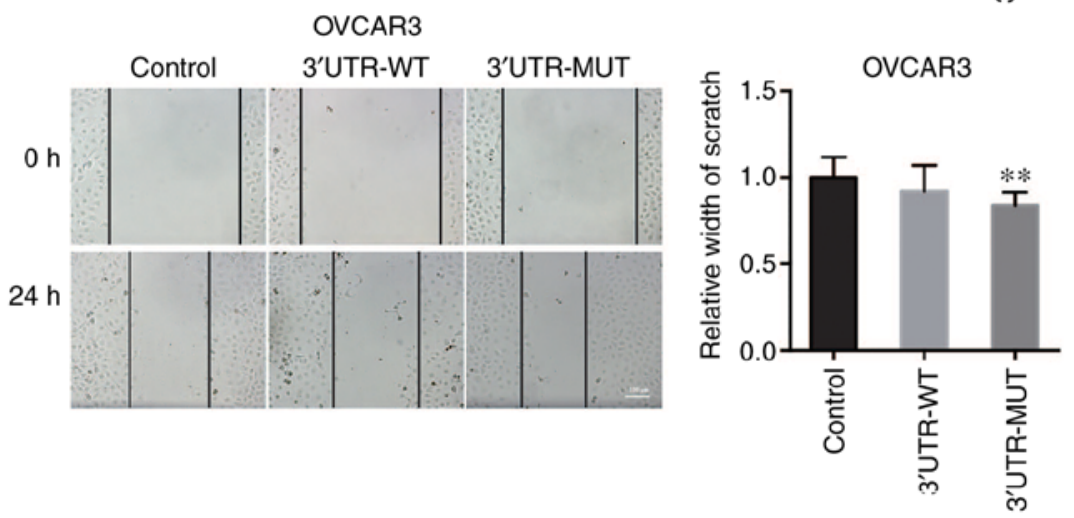
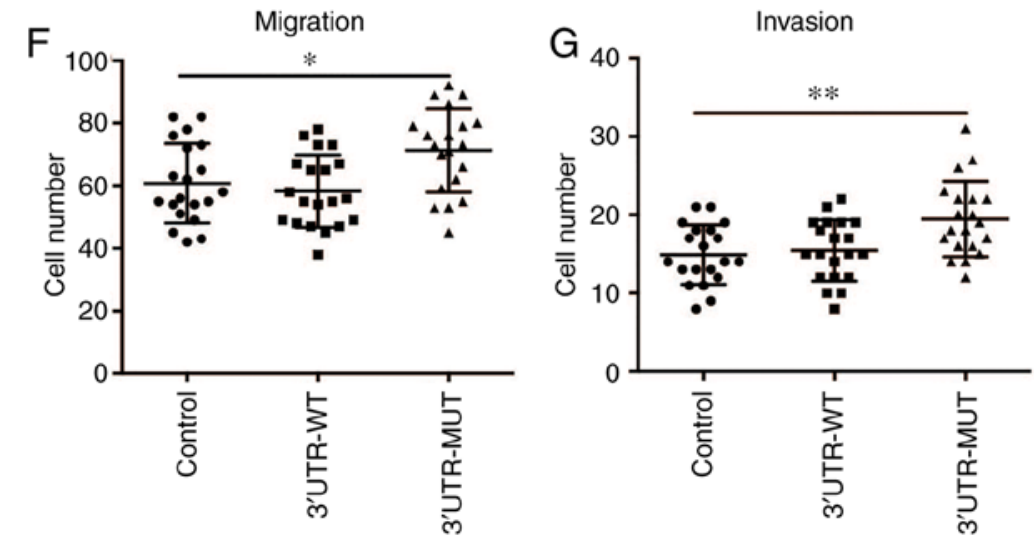

Figure 2. Mutant GAPDH 3'UTR promotes tumor growth and cell motility of OVCA. Cell viability of (A) SKOV3 and (B) OVCAR3 cells stably expressing WT or mutant (MUT) GAPDH 3'UTR was determined by CCK-8 assay. Cells infected with pLKO.1-control lentivirus were used as the negative control. In the CCK-8 assay experiment, 4 parallel wells were set up in each group, and the experiment was repeated three times. Wound healing assays were performed to assess cell migration in (C) SKOV3 and (D) OVCAR3 cells stably expressing WT or MUT GAPDH 3'UTR. Cells infected with pLKO.1-control lentivirus were used as the negative control. $\mathrm{n}=20$. Scale bar in D represents $100 \mu \mathrm{m}$. (E) Cell migration and invasive capacity was examined in SKOV3 cells stably expressing WT or MUT GAPDH 3'UTR using a Transwell assay. SKOV3 cells infected with pLKO.1-control lentivirus were used as the negative control. Scale bar in E represents $100 \mu \mathrm{m}$. Quantification of (F) Transwell migration and (G) Transwell invasion assays, $\mathrm{n}=20$. In the Transwell assay experiment, three parallel wells were set up in each group, and the experiment was repeated three times. ${ }^{*} \mathrm{P}<0.05,{ }^{* *} \mathrm{P}<0.01$, GAPDH, glyceraldehyde 3-phosphate dehydrogenase; UTR, untranslated region; OVCA, ovarian cancer.

Biotech Co., Ltd.) was added at $0,12,24,36,48,60$ or $72 \mathrm{~h}$. The plates were further cultured for $2 \mathrm{~h}$. Cell viability was assessed by measuring the optical density (OD) absorbance at a wavelength of $450 \mathrm{~nm}$ using a microplate reader (BioTek Instruments, Inc.).

Wound healing assays. Cells were cultured to $80 \%$ confluence in 6-well culture plates and a wound was created using a $200-\mu 1$ pipette tip. Subsequently, the cells were washed once with fresh medium to remove the non-adherent cells. Wounds were imaged at 0 and $24 \mathrm{~h}$ and the width of scratch were measured using ImageJ Software (1.48V; NIH).

Cell migration and invasion assays. Cells were resuspended in $200 \mu 1$ serum-free RPMI-1640 medium and were plated onto either a BD BioCoat ${ }^{\mathrm{TM}}$ Matrigel $^{\mathrm{TM}}$ Invasion Chamber (BD Biosciences) for invasion assays, or onto a BD Falcon (uncoated) Chamber for migration assays. For both assays, supplemented medium containing $10 \%$ FBS was added to the lower chamber. The chambers were processed after $24 \mathrm{~h}$ 
A

\begin{tabular}{|lc|}
\hline WT-GAPDH 3'UTR & 5'...TGAAGAGGGGAGGGGCCUAGGGA... \\
hsa-miR-125b & 3' AGUGUUCAAUCCCAGAGUCCCU \\
MUT-GAPDH 3'UTR & 5'...TGAAGAGGGGAGGGGCCUCAGGGA... \\
hsa-miR-125b & 3' AGUGUUCAAUCCCAGAGUCCCU
\end{tabular}

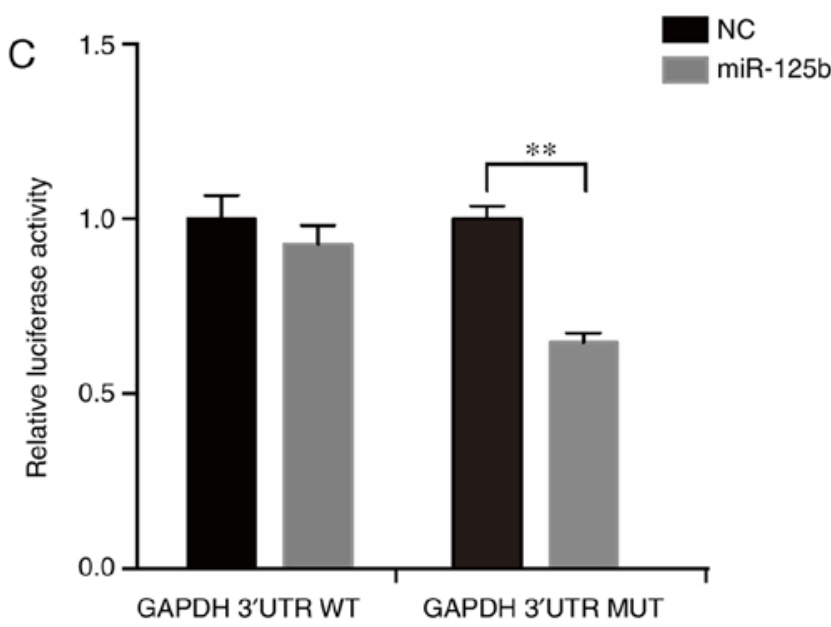

B

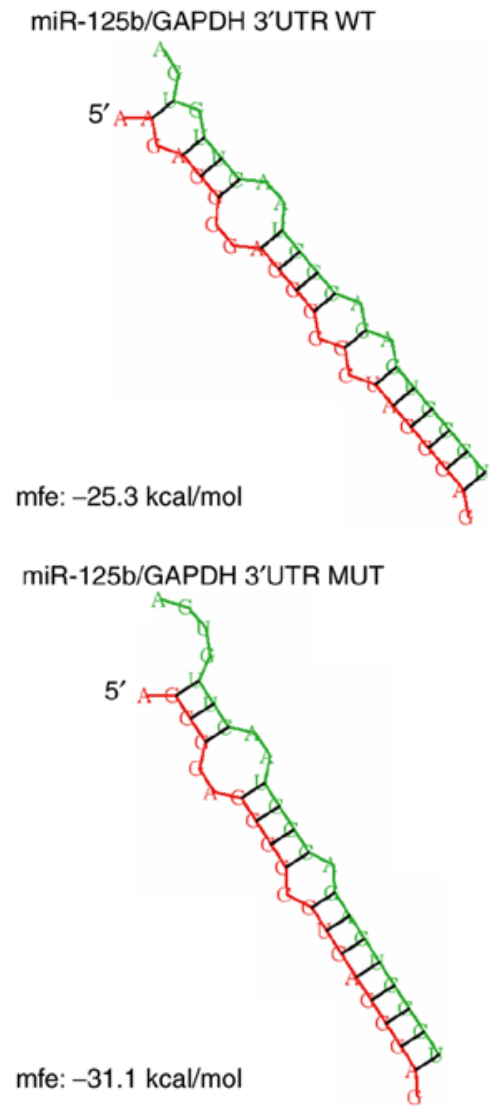

Figure 3. Mutation in GAPDH 3'UTR results in the creation of a miR-125b binding site. (A) Sequence alignment of miR-125b binding site with wild-type (WT) or mutant (MUT) GAPDH 3'UTR. (B) Schematic structure and the free energy of potential binding between miR-125b and WT or MUT GAPDH 3'UTR. (C) miR-125b mimics or scramble NC were co-transfected with luciferase reporter constructs containing WT or MUT GAPDH 3'UTR in 293T cells and relative luciferase activity was measured. GAPDH, glyceraldehyde 3-phosphate dehydrogenase; UTR, untranslated region; NC, negative control; miR, microRNA; mfe, minimum free energy.

according to the manufacturer's protocol, and migrated cells were stained with Hoechst at room temperature for $10 \mathrm{~min}$. Cells were examined using a fluorescence microscope with 20x objective (Nikon Corporation).

microRNA stability analysis. SKOV3 cells were transfected with control WT or mutant GAPDH 3'UTR constructs for $24 \mathrm{~h}$. Subsequently, actinomycin-D was added and cells were further cultured for 4, 8, 12, 16, 20 and $24 \mathrm{~h}$. Total RNA was extracted using TRIzol reagent (Tiangen Biotech Co., Ltd.). The expression of miR-125b was detected using RT-qPCR.

RNA binding immunoprecipitation. Cells were lysed for at least $20 \mathrm{~min}$ on ice. The cell lysates were collected following centrifugation (12,000 x g for $10 \mathrm{~min})$. Cell lysates were incubated with an anti-Ago2 antibody (Abcam; cat. no. ab57113) at a dilution of 1:200, or isotype control IgG (Sigma-Aldrich: Merck $\mathrm{KGaA}$ ) at $4^{\circ} \mathrm{C}$ for $2 \mathrm{~h}$. The RNA-protein immunocomplexes were enriched using protein $\mathrm{A} / \mathrm{G}$ Plus agarose beads. Subsequently, the beads were washed and the complexes were treated with DNase I and Proteinase K. RT-qPCR was performed to assess the RNA isolated from the immunoprecipitation material.

Statistical analysis. Data are presented as the mean \pm standard error of the mean of the indicated number of measurements.
The differences between groups were analyzed by Student's t-test. Comparisons between multiple groups were performed using one-way ANOVA analysis of variance followed by the Tukey's post-hoc test. $\mathrm{P}<0.05$ was considered to indicate a statistically significant difference.

\section{Results}

Identification of a frequent somatic mutation in the 3'UTR of GAPDH. Transcriptome sequencing was performed on the OVCA samples and their matching adjacent normal tissues from 120 patients to analyze the presence and frequency of mutations of housekeeping genes. A summary of the top 10 housekeeping genes with a high mutation rate in the UTR is shown in Fig. 1A. The gene with the highest frequency of non-coding mutations in the UTR was GAPDH, which harbored 6 mutations in 22/120 (18.3\%) OVCA tissues (Table SI). Additionally, a somatic mutation located in GAPDH 3'UTR (NM_001289745.2:c.*1325_*1326insC) occurred most frequently $(9.17 \%)$. In contrast, the matching adjacent normal tissues did not contain this somatic mutation.

The expression of GAPDH in OVCA tissues was assessed using RT-qPCR. As $18 \mathrm{~S}$ and GUSB are reliable reference genes to determine the profile of gene expression in OVCA (29), they were used as the internal controls in the present study. The results showed that GAPDH mRNA levels were significantly 
A

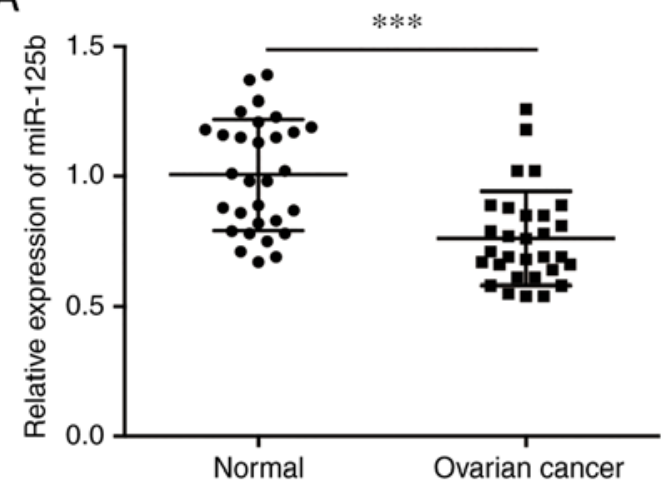

C

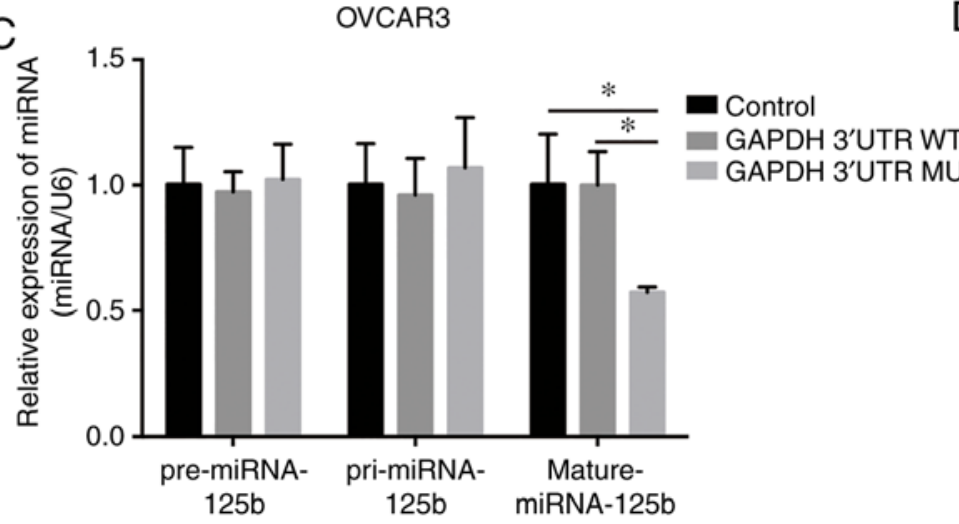

B

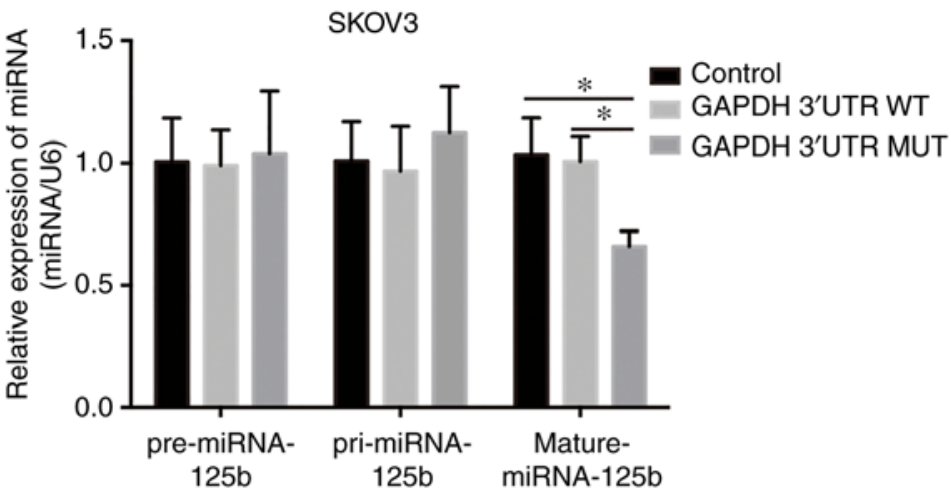

D

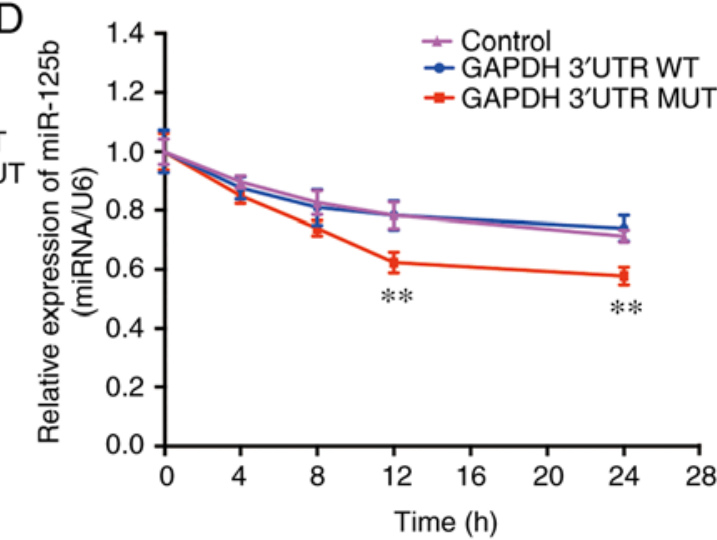

Figure 4. Mutation in GAPDH 3'UTR results in the downregulation of mature miR-125b levels in OVCA cells. (A) mRNA expression levels of miR-125b in 30 paired human OVCA tissues. Detection of pre-miR-125b, pri-miR-125b and mature miR-125b levels in (B) SKOV3 and (C) OVCAR3 cells stably expressing wild-type (WT) or mutant (MUT) GAPDH 3'UTR. Cells infected with pLKO.1-control lentivirus were used as the negative control. (D) Stability assessment of miR-125b in SKOV3 cells transfected with WT or MUT GAPDH 3'UTR constructs. ${ }^{*} \mathrm{P}<0.05,{ }^{* *} \mathrm{P}<0.01,{ }^{* * *} \mathrm{P}<0.001$. GAPDH, glyceraldehyde 3-phosphate dehydrogenase; UTR, untranslated region; OVCA, ovarian cancer; miR, microRNA.

upregulated in OVCA tissues (Fig. 1B). This finding was supported by data from the Oncomine database (oncomine. org/resource/main.html). In contrast to the GAPDH mRNA expression patterns, the expression levels of GAPDH protein did not differ significantly between the cancerous and non-cancerous tissues (Fig. S1).

Mutant GAPDH 3'UTR promotes cell growth and cell motility of OVCA. To determine the biological roles of the effect of the mutant GAPDH 3'UTR (NM_001289745.2:c.*1325_*1326in sC) on OVCA, the OVCA cell lines SKOV3 and OVCAR3 stably expressing WT or mutant GAPDH 3'UTR were constructed. A CCK-8 assay demonstrated that the mutant but not the WT GAPDH 3'UTR-overexpressing OVCA cells exhibited significantly increased cell growth compared with the control cells (Fig. 2A and B). Furthermore, the effect of the GAPDH 3'UTR on cell motility of OVCA cells was assessed using wound healing and Transwell assays. The results showed that overexpression of the mutant GAPDH 3'UTR in OVCA cells significantly promoted migration as the wound closure rate was increased (Fig. 2C and D). Consistent with this result, Transwell assays also showed that the mutant GAPDH 3'UTR significantly increased the migratory and invasive capacity of OVCA cells (Fig. 2E-G). Taken together, these results suggest that mutant GAPDH 3'UTR promoted cell growth and cell motility of OVCA cells.
Mutation in GAPDH 3'UTR results in the creation of a miR-125b binding site. To further analyze the molecular mechanisms of the frequent mutation in GAPDH 3'UTR, bioinformatics algorithms were used to predict miRNAs which may interact with the mutation site, including miRDB (30) and miRcode (31). The results of the alignment analysis suggested that the mutated sequence matches a sequence located in the 'seed region' of the binding sites of miR-125b (Fig. 3A). The structure and free energy of potential binding site interactions between miR-125b with GAPDH mRNA 3'UTR (WT and mutant) were analyzed using RNAhybrid (32). The results showed that the mutant GAPDH 3'UTR had a stronger affinity for miR-125b compared with the WT (Fig. 3B). To further confirm the binding activation of GAPDH 3'UTR with miR-125b, luciferase reporter plasmids with the WT or mutant GAPDH 3'UTR were constructed and co-transfected with miR-125b mimics in $293 \mathrm{~T}$ cells. The results showed that only when transfected with the mutant construct was the activity of luciferase significantly reduced by miR-125b (Fig. 3C). Based on these results, it was concluded that the frequent mutation in GAPDH 3'UTR created a new binding site for miR-125b.

Mutation in GAPDH 3'UTR results in the downregulation of mature miR-125b levels in OVCA cells. Previous studies have shown that miR-125b is dysregulated in different types of 

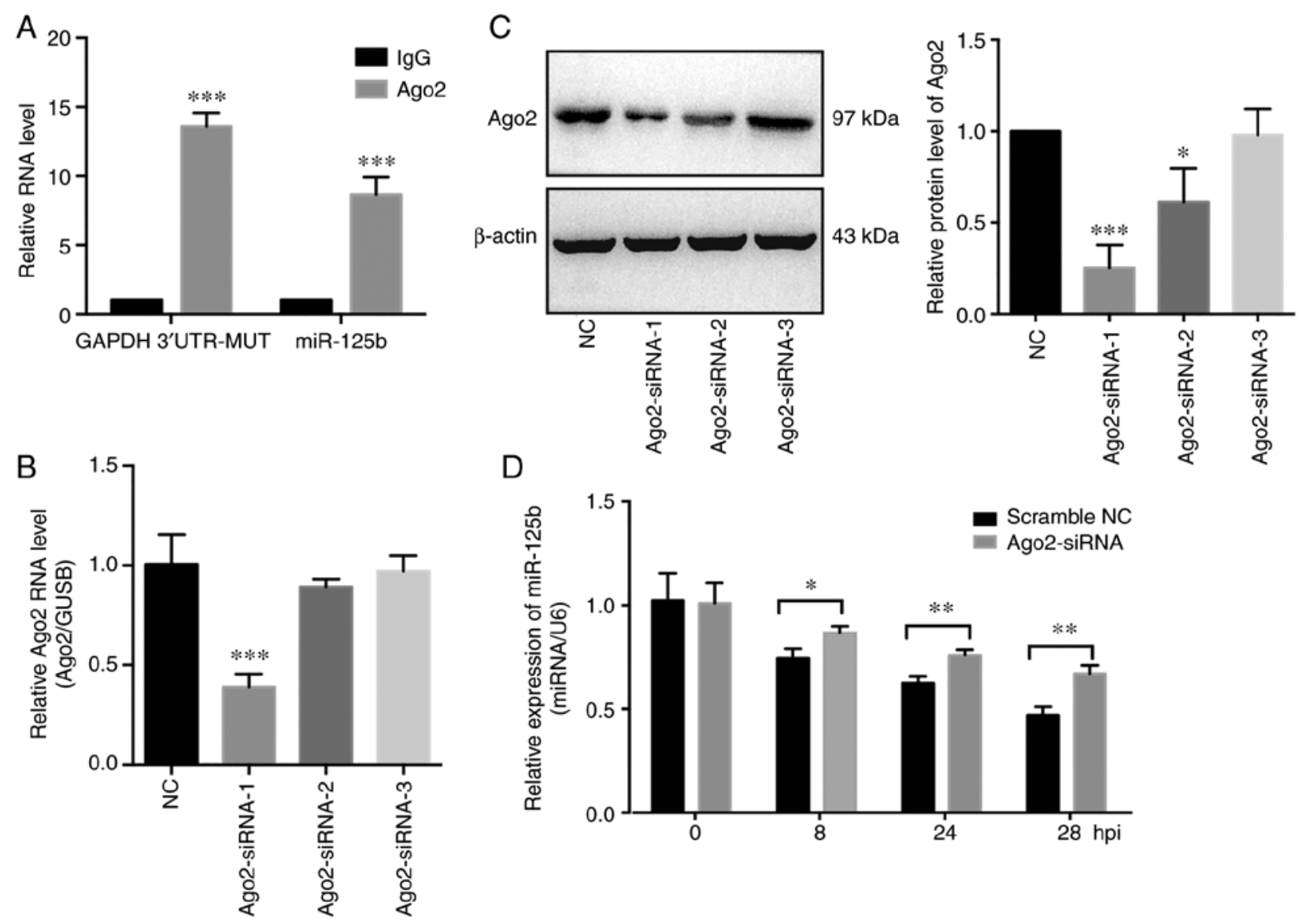

Figure 5. Mutant GAPDH 3'UTR regulates miR-125b levels in an Ago2-dependent manner. (A) RNA immunoprecipitation (RIP) assays were performed and the relative enrichment of the mutant (MUT) GAPDH 3'UTR RNA and miR-125b were determined using RT-qPCR in the Ago2 and IgG groups. (B) RT-qPCR and (C) western blot analysis were used to assess the efficiency of Ago2 siRNA; GUSB and $\beta$-actin were used as the internal controls, respectively. Ago2-siRNA-1 was the most effective siRNA and thus used for subsequent experiments. (D) Assessment of the stability of miR-125b in SKOV3 cells transfected with the mutant GAPDH 3'UTR construct and Ago2 siRNA-1; scramble NC was used as the negative control. GAPDH, glyceraldehyde 3-phosphate dehydrogenase; RT-qPCR, reverse transcription-quantitative PCR; siRNA, small interfering RNA; UTR, untranslated region; NC, negative control; Ago2, argonaute RISC catalytic component $2 .{ }^{*} \mathrm{P}<0.05,{ }^{* *} \mathrm{P}<0.01,{ }^{* * * *} \mathrm{P}<0.001$.

cancer (33-36). The expression of miR-125b was quantified in 30 paired normal and OVCA tissues using RT-qPCR, and it was found that miR-125b was significantly downregulated in the OVCA tissues (Fig. 4A). This finding is supported by several recent studies $(33,37)$. Additionally, miR-125b levels may have been regulated by the mutant GAPDH 3'UTR in OVCA cells. Expression of the mutant GAPDH 3'UTR significantly decreased the levels of mature miR-125b, whereas neither pre-miRNA nor pri-miRNA levels were altered (Fig. 4B and C); and the stability of mature miR-125b was significantly decreased in cells stably expressing the mutant GAPDH 3'UTR following inhibition of intracellular RNA transcription with actinomycin D treatment (Fig. 4D). These phenomena were abrogated in cells with the WT 3'UTR of GAPDH (Fig. 4B-D). Additionally, the mature miR-125b level in the OVCA mutant tissues (NM_001289745.2:c.*13 25_*1326insC) and OVCA wild-type tissues was analyzed. The results showed there was no difference between these two group, and the main reason was the low abundance of GAPDH mutation (Fig. S2). Together, these results suggest that the mutant GAPDH 3'UTR reduced the relative levels of mature miR-125b, and this effect was not associated with transcriptional regulation or miRNA precursor processing.
Mutant GAPDH 3'UTR regulates miR-125b levels in an Ago2-dependent manner. It was previously reported that miRNAs guide argonaute RISC catalytic component 2 (Ago2) protein to distinct target sites on mRNAs (38). Therefore, the involvement of Ago2 on the interaction of miR-125b and GAPDH 3'UTR-MUT was assessed. Subsequently, to further explore the potential mechanisms involved, an anti-Ago2 RIP experiment was performed on extracts of SKOV3 cells (stably expressing mutant GAPDH 3'UTR). The mutant GAPDH 3'UTR fragment and miR-125b were enriched in Ago2-containing complexes (Fig. 5A). In addition, to investigate the role of Ago2 on the biological function of the mutant GAPDH 3'UTR, Ago2 expression was knocked down using siRNA (Fig. 5B and C). Knockdown of Ago2 partially inhibited the degradation of miR-125b induced by the mutant GAPDH 3'UTR (Fig. 5D). Previous studies showed that miRNAs act as miRNA ribonucleoprotein complexes containing Ago2, an important component of RNA induced silencing complex (RISC). The above results showed that miR-125b and the mutant GAPDH 3'UTR were involved in a RISC, suggesting that the mutant GAPDH 3'UTR may function as a sponge of miR-125b in OVCA cells. 
A

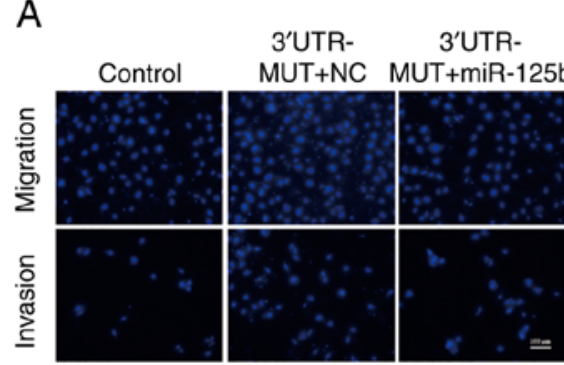

B

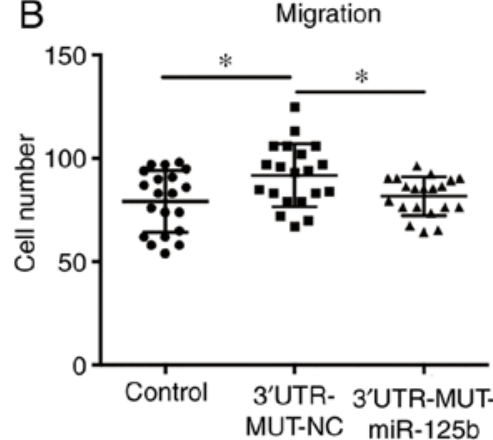

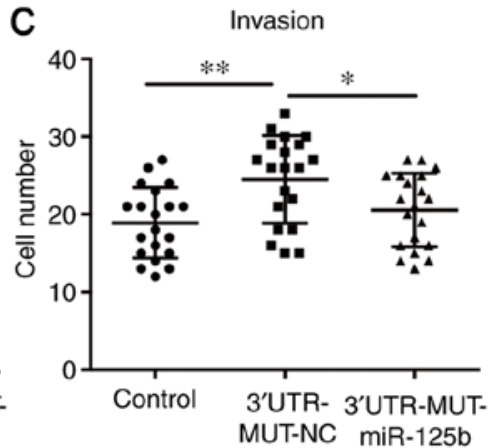

$\mathrm{D}$

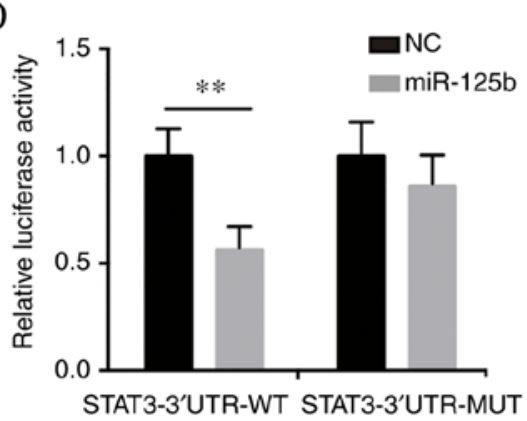

$\mathrm{E}$

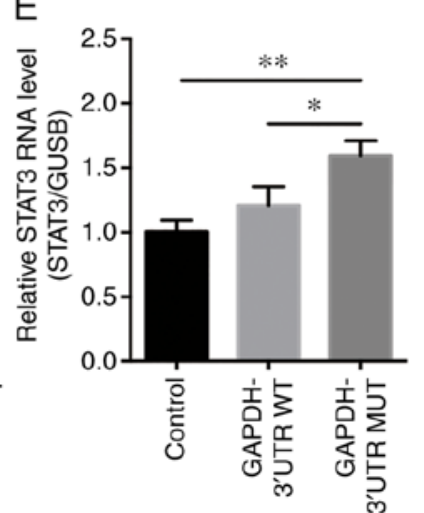

$\mathrm{F}$

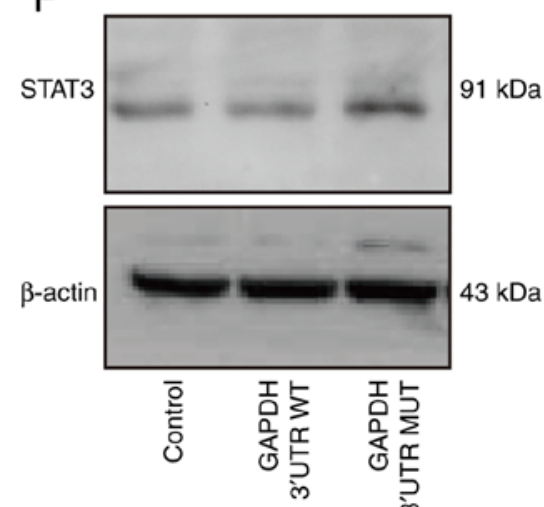

Figure 6. Mutant GAPDH 3'UTR promotes cancer development by sponging miR-125b, thereby regulating STAT3 expression in OVCA cells. (A) miR-125b mimics or scramble NC were transfected into SKOV3 cells stably expressing the mutant (MUT) GAPDH 3'UTR. Migration and invasion assays were performed to assess the effects of miR-125b on cell motility. SKOV3 cells infected with pLKO.1-control lentivirus were used as the negative control. Scale bar in A represents $100 \mu \mathrm{m}$. Quantification of (B) migration and (C) invasion, $\mathrm{n}=20$. (D) Luciferase reporter assays showed that overexpression of miR-125b reduced luciferase activity in 293T cells transfected with the STAT3 3'UTR-WT vector. (E) RT-qPCR and (F) western blot analysis of STAT3 expression in SKOV3 cells stably expressing WT of mutant GAPDH 3'UTR. SKOV3 cells infected with pLKO.1-control lentivirus were used as the negative control. "P<0.05, ${ }^{* *} \mathrm{P}<0.01$. GAPDH, glyceraldehyde 3-phosphate dehydrogenase; STAT3, signal transducer and activator of transcription 3; UTR, untranslated region; OVCA, ovarian cancer; RT-qPCR, reverse transcription-quantitative PCR; miR, microRNA; NC, negative control.

Mutant GAPDH 3'UTR promotes cancer development by sponging miR-125b, thereby regulating STAT3 expression in OVCA cells. To investigate whether the effects of the mutant GAPDH 3'UTR on OVCA development were mediated by miR-125b, OVCA cells stably expressing the mutant GAPDH 3'UTR were transfected with miR-125b mimics. Transfection of miR-125b mimics abrogated the tumor promoting effects induced by mutant GAPDH 3'UTR, which suggested that the mutant GAPDH 3'UTR promoted cancer development by regulating miR-125b (Fig. 6A-C). To examine the effects of miR-125b on the mechanisms and functions of the mutant GAPDH 3'UTR, direct target genes of miR-125b were selected using TargetScan. The results showed that STAT3 may be an effective target gene. Luciferase reporter assays were performed to assess whether STAT3 directly interacted with miR-125b. The results demonstrated that miR-125b reduced luciferase activity in the pGL3-STAT3-3'UTR-WT transfected cells (Fig. 6D). To determine the underlying relationship between GAPDH 3'UTR and STAT3, the expression of STAT3 was assessed in SKOV3 cells stably expressing GAPDH 3'UTR. The results showed that overexpression of the mutant GAPDH 3'UTR upregulated STAT3 expression (Fig. 6E and F). As it has been reported that elevated STAT3 expression in OVCA contributes to tumorigenesis and progression $(39,40)$, it was concluded that the mutant GAPDH 3'UTR facilitates OVCA cell growth and cell motility by sponging miR-125b and thus upregulating STAT3 levels.

\section{Discussion}

Recently, the genomic sequencing of cancer tissues has provided evidence that the majority of cancer genomes contain thousands of somatic mutations, and some of these somatic mutations may exhibit a functional effect on tumorigenesis and metastasis $(16,41,42)$. In the present study, GAPDH mRNA expression levels were upregulated in ovarian cancer (OVCA) tissues, and a frequent somatic mutation in the $3^{\prime}$ UTR of GAPDH (NM_001289745.2:c.*1325_*1326insC) was identified. The mutant GAPDH 3'UTR significantly promoted OVCA cell growth and cell motility, whereas the WT GAPDH 3'UTR had no effect on these biological processes.

Numerous studies have shown that somatic mutations in the $3^{\prime}$ UTR may promote tumor progression by altering miRNA targeting efficiency and consequently affecting miRNA-mRNA interactions $(18,43,44)$. To assess the functional mechanisms of a frequent somatic mutation located in the 3'UTR of GAPDH, its effect on miRNA binding was analyzed. The results showed that the mutation created a new binding site for miR-125b. RT-qPCR was performed on OVCA cell lines stably expressing WT or mutant GAPDH 3'UTR, and the results showed that the mutant 3'UTR resulted in a $\sim 40 \%$ decrease in mature miR-125b levels, and this regulation was dependent on the expression of Ago2. These results suggest that the GAPDH 3'UTR mutation, which is able to bind to miR-125b target sites, may impair miRNA-mRNA interaction and sponge miR-125b. 
It was reported that miR-125b was involved in the initiation and progression of several types of cancer, such as urothelial tumors (45), liver $(46,47)$, bladder cancer (48) and ovarian cancer (33). miR-125b may act as a tumor suppressor, inhibiting invasion, migration and angiogenesis $(48,49)$. In the present study, miR-125b was downregulated in OVCA tissues compared with the matching normal tissues. Transfection of miR-125b mimics suppressed OVCA cell growth, and decreased cell motility. In addition, transfection of miR-125b mimics counteracted the tumor promoting effects induced by the mutant GAPDH 3'UTR. Taken together, these results indicated that the mutant GAPDH 3'UTR promoted the development of OVCA by sponging miR-125b.

Several recent studies have reported that mRNAs, transcribed pseudogenes and other RNAs may bind to miRNAs and act as potent miRNA sponges (50-52). These molecules have been termed competitive endogenous RNAs (20). ceRNAs can sequester miRNAs, thereby protecting the target RNAs of the respective miRNA from repression (23). In the present study, the mutant GAPDH 3'UTR regulated miR-125b levels in an Ago2-dependent manner, which suggested that the mutant GAPDH may act as a ceRNA. Bioinformatics algorithms and a luciferase reporter assay identified STAT3 as the target of miR-125b. Moreover, this finding was supported by several recent studies $(53,54)$. To further determine the effect of the mutant GAPDH 3'UTR on miR-125b-mediated regulation of STAT3, the expression levels of STAT3 in OVCA cells stably expressing GAPDH 3'UTR were assessed. Overexpression of the mutant GAPDH 3'UTR resulted in a significant increase in STAT3 levels. Several studies have shown that STAT3 levels are elevated in several different types of cancer, and it is a critical regulator of numerous cellular processes, including proliferation, invasion and metastasis (55-57). Therefore, the mutant GAPDH 3'UTR may promote tumor growth and cell motility through increasing the expression of STAT3.

In conclusion, the primary finding of the present study was that mutations in GAPDH 3'UTR promoted development of OVCA by creating a new miR-125b binding site, thereby promoting oncogenic STAT3 expression in ovarian cancer.

\section{Acknowledgements}

We would like to thank the Research Fund for Academician Lin He New Medicine for the financial support of this study, and Genex Health Co., Ltd. (Beijing, China) for their assistance during the preparation of this manuscript.

\section{Funding}

The study was supported by grants from Research Fund for Academician Lin He New Medicine.

\section{Availability of data and materials}

The datasets used and/or analyzed during the present study are available from the corresponding author on reasonable request.

\section{Authors' contributions}

PL, YZ and $\mathrm{HH}$ contributed to the design of this study. PL, YZ, $\mathrm{TC}, \mathrm{XS}$ and $\mathrm{HH}$ performed the experiments, and analyzed and interpreted the data. PL, YZ and $\mathrm{HH}$ wrote the manuscript. XS revised the manuscript. All authors read and approved the manuscript and agree to be accountable for all aspects of the research in ensuring that the accuracy or integrity of any part of the work are appropriately investigated and resolved.

\section{Ethics approval and consent to participate}

The study was approved by the Ethics Committee of The Third Affiliated Hospital of Guangzhou Medical University (Guangzhou, Guangdong, China), and written informed consent was obtained from all patients.

\section{Patient consent for publication}

Not applicable.

\section{Competing interests}

The authors declare that they have no competing interests.

\section{References}

1. Bray F, Ferlay J, Soerjomataram I, Siegel RL, Torre LA and Jemal A: Global cancer statistics 2018: GLOBOCAN estimates of incidence and mortality worldwide for 36 cancers in 185 countries. CA Cancer J Clin 68: 394-424, 2018.

2. Torre LA, Bray F, Siegel RL, Ferlay J, Lortet-Tieulent J and Jemal A: Global cancer statistics, 2012. CA Cancer J Clin 65: 87-108, 2015.

3. Siegel RL, Miller KD and Jemal A: Cancer statistics, 2015. CA Cancer J Clin 65: 5-29, 2015.

4. Glaser PE and Gross RW: Rapid plasmenylethanolamine-selective fusion of membrane bilayers catalyzed by an isoform of glyceraldehyde-3-phosphate dehydrogenase: Discrimination between glycolytic and fusogenic roles of individual isoforms. Biochemistry 34: 12193-12203, 1995.

5. Tisdale EJ, Kelly C and Artalejo CR: Glyceraldehyde-3-phosphate dehydrogenase interacts with Rab2 and plays an essential role in endoplasmic reticulum to Golgi transport exclusive of its glycolytic activity. J Biol Chem 279: 54046-54052, 2004

6. Hara MR and Snyder SH: Nitric oxide-GAPDH-Siah: A novel cell death cascade. Cell Mol Neurobiol 26: 527-538, 2006.

7. Baxi MD and Vishwanatha JK: Uracil DNA-glycosylase/glyceraldehyde-3-phosphate dehydrogenase is an Ap4A binding protein. Biochemistry 34: 9700-9707, 1995.

8. Zheng L, Roeder RG and Luo Y: S phase activation of the histone $\mathrm{H} 2 \mathrm{~B}$ promoter by OCA-S, a coactivator complex that contains GAPDH as a key component. Cell 114: 255-266, 2003.

9. Barroso I, Benito B, Garcí-Jiménez C, Hernández A, Obregón MJ and Santisteban P: Norepinephrine, tri-iodothyronine and insulin upregulate glyceraldehyde-3-phosphate dehydrogenase mRNA during Brown adipocyte differentiation. Eur J Endocrinol 141: 169-179, 1999.

10. Valenti MT, Bertoldo F, Dalle Carbonare L, Azzarello G, Zenari S, Zanatta M, Balducci E, Vinante O and Lo Cascio V: The effect of bisphosphonates on gene expression: GAPDH as a housekeeping or a new target gene? BMC Cancer 6: 49, 2006.

11. Sikand K, Singh J, Ebron JS and Shukla GC: Housekeeping gene selection advisory: Glyceraldehyde-3-phosphate dehydrogenase (GAPDH) and $\beta$-actin are targets of miR-644a. PLoS One 7: e47510, 2012

12. Bjerregaard H, Pedersen S, Kristensen SR and Marcussen N: Reference genes for gene expression analysis by real-time reverse transcription polymerase chain reaction of renal cell carcinoma. Diagn Mol Pathol 20: 212-217, 2011.

13. Ma Y, Dai H, Kong X and Wang L: Impact of thawing on reference gene expression stability in renal cell carcinoma samples. Diagn Mol Pathol 21: 157-163, 2012.

14. Majidzadeh-A K, Esmaeili R and Abdoli N: TFRC and ACTB as the best reference genes to quantify Urokinase plasminogen activator in breast cancer. BMC Res Notes 4: 215, 2011. 
15. Guo Y, Chen JX, Yang S, Fu XP, Zhang Z, Chen KH, Huang Y, Li Y, Xie Y and Mao YM: Selection of reliable reference genes for gene expression study in nasopharyngeal carcinoma. Acta Pharmacol Sin 31: 1487-1494, 2010.

16. Stratton MR: Exploring the genomes of cancer cells: Progress and promise. Science 331: 1553-1558, 2011.

17. Nicoloso MS, Sun H, Spizzo R, Kim H, Wickramasinghe P, Shimizu M, Wojcik SE, Ferdin J, Kunej T, Xiao L, et al: Single-nucleotide polymorphisms inside microRNA target sites influence tumor susceptibility. Cancer Res 70: 2789-2798, 2010.

18. Wang Y, Du X, Zhou Z, Jiang J, Zhang Z, Ye L and Hong H: A gain-of-function ACTC1 3'UTR mutation that introduces a miR-139-5p target site may be associated with a dominant familial atrial septal defect. Sci Rep 6: 25404, 2016.

19. Flynt AS andLai EC: Biological principles of microRNA-mediated regulation: Shared themes amid diversity. Nat Rev Genet 9: 831-842, 2008

20. Salmena L, Poliseno L, Tay Y, Kats L and Pandolfi PP: A ceRNA hypothesis: The Rosetta stone of a hidden RNA language? Cell 146: 353-358, 2011.

21. Tay Y, Rinn J and Pandolfi PP: The multilayered complexity of ceRNA crosstalk and competition. Nature 505: 344-352, 2014.

22. Fang L, Du WW, Yang X, Chen K, Ghanekar A, Levy G, Yang W, Yee AJ, Lu WY, Xuan JW, et al: Versican 3'-untranslated region (3'-UTR) functions as a ceRNA in inducing the development of hepatocellular carcinoma by regulating miRNA activity. FASEB J 27: 907-919, 2013

23. Karreth FA, Tay Y, Perna D, Ala U, Tan SM, Rust AG, DeNicola G Webster KA, Weiss D, Perez-Mancera PA, et al: In vivo identification of tumor-suppressive PTEN ceRNAs in an oncogenic BRAF-induced mouse model of melanoma. Cell 147: 382-395, 2011

24. Lee DY, Shatseva T, Jeyapalan Z, Du WW, Deng Z and Yang BB A 3'-untranslated region (3'UTR) induces organ adhesion by regulating miR-199a* functions. PLoS One 4: e4527, 2009.

25. Sumazin P, Yang X, Chiu HS, Chung WJ, Iyer A, Llobet-Navas D, Rajbhandari P, Bansal M, Guarnieri P, Silva J and Califano A: An extensive microRNA-mediated network of RNA-RNA interactions regulates established oncogenic pathways in glioblastoma. Cell 147: 370-381, 2011

26. Calin GA and Croce CM: MicroRNA signatures in human cancers. Nat Rev Cancer 6: 857-866, 2006.

27. Croce CM: Causes and consequences of microRNA dysregulation in cancer. Nat Rev Genet 10: 704-714, 2009.

28. Stahlhut C and Slack FJ: MicroRNAs and the cancer phenotype: Profiling, signatures and clinical implications. Genome Med 5: $111,2013$.

29. Li YL, Ye F, Hu Y, Lu WG and Xie X: Identification of suitable reference genes for gene expression studies of human serous ovarian cancer by real-time polymerase chain reaction. Anal Biochem 394: 110-116, 2009.

30. Wang J, Yu H, Ye L, Jin L, Yu M and Lv Y: Integrated regulatory mechanisms of miRNAs and targeted genes involved in colorectal cancer. Int J Clin Exp Pathol 8: 517-529, 2015.

31. Jeggari A, Marks DS and Larsson E: miRcode: A map of putative microRNA target sites in the long non-coding transcriptome. Bioinformatics 28: 2062-2063, 2012.

32. Rehmsmeier M, Steffen P, Hochsmann M and Giegerich R: Fast and effective prediction of microRNA/target duplexes. RNA 10 $1507-1517,2004$

33. Ying X, Wei K, Lin Z, Cui Y, Ding J, Chen Y and Xu B: MicroRNA-125b suppresses ovarian cancer progression via suppression of the epithelial-mesenchymal transition pathway by targeting the SET protein. Cell Physiol Biochem 39: 501-510, 2016.

34. Vriens MR, Weng J, Suh I, Huynh N, Guerrero MA, Shen WT, Duh QY, Clark OH and Kebebew E: MicroRNA expression profiling is a potential diagnostic tool for thyroid cancer. Cancer 118: 3426-3432, 2012.

35. Jia HY, Wang YX, Yan WT, Li HY, Tian YZ, Wang SM and Zhao HL: MicroRNA-125b functions as a tumor suppressor in hepatocellular carcinoma cells. Int J Mol Sci 13: 8762-8774, 2012

36. Henson BJ, Bhattacharjee S, O'Dee DM, Feingold E and Gollin SM: Decreased expression of miR-125b and miR-100 in oral cancer cells contributes to malignancy. Genes Chromosomes Cancer 48: 569-582, 2009.

37. Iorio MV, Visone R, Di Leva G, Donati V, Petrocca F, Casalini P, Taccioli C, Volinia S, Liu CG, Alder H, et al: MicroRNA signatures in human ovarian cancer. Cancer Res 67: 8699-8707, 2007.

38. Meister G, Landthaler M, Patkaniowska A, Dorsett Y, Teng G and Tuschl T: Human Argonaute 2 mediates RNA cleavage targeted by miRNAs and siRNAs. Mol Cell 15: 185-197, 2004.
39. Saini U, Naidu S, ElNaggar AC, Bid HK, Wallbillich JJ, Bixel K, Bolyard C, Suarez AA, Kaur B, Kuppusamy P, et al: Elevated STAT3 expression in ovarian cancer ascites promotes invasion and metastasis: A potential therapeutic target. Oncogene 36: 168-181, 2017.

40. Wen W, Liang W, Wu J, Kowolik CM, Buettner R, Scuto A, Hsieh MY, Hong H, Brown CE, Forman SJ, et al: Targeting JAK1/STAT3 signaling suppresses tumor progression and metastasis in a peritoneal model of human ovarian cancer. Mol Cancer Ther 13: 3037-3048, 2014.

41. Boehm JS and Hahn WC: Towards systematic functional characterization of cancer genomes. Nat Rev Genet 12: 487-498, 2011.

42. Chin L, Andersen JN and Futreal PA: Cancer genomics: From discovery science to personalized medicine. Nat Med 17: 297-303, 2011.

43. Wu W, Wu L, Zhu M, Wang Z, Wu M, Li P, Nie Y, Lin X, Hu J, Eskilsson E, et al: miRNA mediated noise making of 3'UTR mutations in cancer. Genes (Basel) 9: pii: E545, 2018.

44. Lopes-Ramos CM, Barros BP, Koyama FC, Carpinetti PA, Pezuk J, Doimo NTS, Habr-Gama A, Perez RO and Parmigiani RB: E2F1 somatic mutation within miRNA target site impairs gene regulation in colorectal cancer. PLoS One 12: e0181153, 2017.

45. Veerla S, Lindgren D, Kvist A, Frigyesi A, Staaf J, Persson H, Liedberg F, Chebil G, Gudjonsson S, Borg A, et al: MiRNA expression in urothelial carcinomas: Important roles of miR-10a, miR-222, miR-125b, miR-7 and miR-452 for tumor stage and metastasis, and frequent homozygous losses of miR-31. Int J Cancer 124: 2236-2242, 2009.

46. Alpini G, Glaser SS, Zhang JP, Francis H, Han Y, Gong J, Stokes A, Francis T, Hughart N, Hubble L, et al: Regulation of placenta growth factor by microRNA-125b in hepatocellular cancer. J Hepatol 55: 1339-1345, 2011.

47. Zhou JN, Zeng Q, Wang HY, Zhang B, Li ST, Nan X, Cao N, Fu CJ, Yan XL, Jia YL, et al: MicroRNA-125b attenuates epithelial-mesenchymal transitions and targets stem-like liver cancer cells through small mothers against decapentaplegic 2 and 4. Hepatology 62: 801-815, 2015.

48. Han Y, Liu Y, Zhang H, Wang T, Diao R, Jiang Z, Gui Y and Cai Z: Hsa-miR-125b suppresses bladder cancer development by down-regulating oncogene SIRT7 and oncogenic long non-coding RNA MALAT1. FEBS Lett 587: 3875-3882, 2013.

49. Xu N, Zhang L, Meisgen F, Harada M, Heilborn J, Homey B, Grandér D, Ståhle M, Sonkoly E and Pivarcsi A: MicroRNA-125b down-regulates matrix metallopeptidase 13 and inhibits cutaneous squamous cell carcinoma cell proliferation, migration, and invasion. J Biol Chem 287: 29899-29908, 2012.

50. Cesana M, Cacchiarelli D, Legnini I, Santini T, Sthandier O, Chinappi M, Tramontano A and Bozzoni I: A long noncoding RNA controls muscle differentiation by functioning as a competing endogenous RNA. Cell 147: 358-369, 2011.

51. Tay Y, Kats L, Salmena L, Weiss D, Tan SM, Ala U, Karreth F, Poliseno L, Provero P, Di Cunto F, et al: Coding-independent regulation of the tumor suppressor PTEN by competing endogenous mRNAs. Cell 147: 344-357, 2011.

52. Poliseno L, Salmena L, Zhang J, Carver B, Haveman WJ and Pandolfi PP: A coding-independent function of gene and pseudogene mRNAs regulates tumour biology. Nature 465: $1033-1038,2010$

53. Jin XJ, Chen XJ, Zhang ZF, Hu WS, Ou RY, Li S, Xue JS, Chen LL, Hu Y and Zhu H: Long noncoding RNA SNHG12 promotes the progression of cervical cancer via modulating miR-125b/STAT3 axis. J Cell Physiol 234: 6624-6632, 2019.

54. Liu LH, Li H, Li JP, Zhong H, Zhang HC, Chen J and Xiao T: miR-125b suppresses the proliferation and migration of osteosarcoma cells through down-regulation of STAT3. Biochem Biophys Res Commun 416: 31-38, 2011.

55. Bromberg J and Darnell JE Jr: The role of STATs in transcriptional control and their impact on cellular function. Oncogene 19: 2468-2473, 2000.

56. Darnell JE: Validating Stat3 in cancer therapy. Nat Med 11: 595-596, 2005

57. Levy DE and Darnell JE Jr: Stats: Transcriptional control and biological impact. Nat Rev Mol Cell Biol 3: 651-662, 2002.

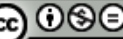

This work is licensed under a Creative Commons Attribution-NonCommercial-NoDerivatives 4.0 International (CC BY-NC-ND 4.0) License. 